Wales, and the Scottish Adolescents Lifestyle and Substance Use Surveys between 1998 and 2015, including 247,515 13 and 15 year-old respondents. Segmented regression analyses examined trends for smoking prevalence (ever smoking and weekly smoking) and perceived smoking norms, testing for change in trend from 2011-15. Falsifiability checks examined change in trends for alcohol use and cannabis use for the same period.

Results In final models for the whole sample, there was no change in rate of decline for ever smoking $(\mathrm{OR}=1.01$; CI 0.99 to 1.03 ), with a marginally significant slowing in the rate of decline for weekly smoking (OR=1.04; CI 1.00 to 1.08). This slowing decline in weekly smoking was however limited to groups for whom rates were declining prior to 2010 at a rate which could not be sustained (i.e. girls and 13 year olds), and occurred in a greater magnitude for other substances, including cannabis use $(\mathrm{OR}=1.21$, CI 1.18 to 1.25$)$ and alcohol use $(\mathrm{OR}=1.17$; CI 1.14 to 1.19$)$. There was consistent evidence across all subgroups of an increased rate of decline in the percentage of young people saying that smoking is ok $(\mathrm{OR}=0.83$; CI 0.81 to 0.86$)$.

Conclusion We found no evidence that the growing prevalence of e-cigarette use has led to increased experimentation with smoking, and some evidence that young people's perceptions against smoking as a normative behavior have hardened rather than softened. Although the decline in weekly smoking rates is slowing, this appears to reflect a floor effect and is of smaller magnitude than change in trend for other substances. While the idea that e-cigarettes renormalize smoking has been central to much policy debate surrounding regulation, these findings indicate no reason to believe that this renormalization is occurring.

\section{OP60 DIFFUSION OF THE ASSIST SMOKING PREVENTION PROGRAMME IN ADOLESCENT SOCIAL NETWORKS BEYOND THE SCHOOL SETTING}

${ }^{1} \mathrm{~F}$ Dobbie*, 'L Bauld, ${ }^{2} \mathrm{~A}$ Amos, ${ }^{1} \mathrm{~S}$ Haw. ${ }^{1}$ Faculty of Health Sciences and Sport, University of Stirling, Stirling, UK; ${ }^{2}$ Usher Institute of Population Health Sciences and Informatics, University of Edinburgh, Edinburgh, UK

\subsection{6/jech-2018-SSMabstracts.59}

Background Smoking prevention programmes that aim to reach adolescents before they start to experiment with tobacco may contribute to reducing tobacco use. ASSIST (A Stop Smoking in Schools Trial) is a peer- led, school and social network based, smoking prevention programme that encourages the dissemination of non-smoking messages among 12-13 year olds in the UK. To date, evaluation of ASSIST has focused on delivery within schools only. This study sought to address this gap by using qualitative social network research to map the social networks of peer supporters to explore the extent, nature, content and perceived impact of message diffusion beyond the school year.

Methods Qualitative, face to face interviews conducted in school with 16 young people aged 12-13 incorporating the creation of 'egocentric sociograms' to collect network data. Sociograms were then used to create a qualitative narrative to explore the extent, and perceived impact, of message diffusion in the wider social networks of peer supporters. Computer packages UCINET and NVivo were used to conduct analysis.
Results Peer supporter networks were fairly large with a total of 155 people. Network composition was weighted slightly more toward family members than friends. Peer supporters recalled having conversations about smoking with 103 people. Over half $(53 \% \mathrm{n}=55)$ of these conversations were with people out with their school year (e.g. parents, siblings, other family members, family friends and neighbours). Thematic analysis of conversation content revealed three types of conversation: protecting non-smokers from starting to smoke; encouraging smokers to stop; and protecting people in wider social networks. Perceived impact was noted for 37 people in peer supporter networks, with examples of positive and negative impact, focusing on the dynamics of a child speaking to an adult.

Conclusion Smoking prevention message diffusion was not limited to school year, reaching in to the wider networks of peer supporters. This creates learning opportunities for the future delivery of ASSIST or other similar peer-led interventions for smoking prevention, and the role of social network interventions in prevention.

\section{Mental health}

\section{OP61 CHRONIC INFLAMMATION AND SUBSEQUENT DEPRESSIVE SYMPTOMS: THE MEDIATING ROLE OF PHYSICAL ACTIVITY}

P Frank* , D Cadar, A Khaushal. Department of Behavioural Science and Health, University College London, London, UK

\subsection{6/jech-2018-SSMabstracts.60}

Background Chronic inflammation has been associated with the onset of depression, but the mechanisms underlying this relationship remain elusive. This study examined whether physical activity (PA) explained the association between elevated levels of inflammatory markers and subsequent depressive symptoms in an English nationally representative sample.

Methods The sample consisted of 2953 men and women (aged 50+) recruited from the English Longitudinal Study of Ageing (ELSA) an ongoing, open, representative prospective cohort study. Four waves of data between 2008/09 (wave 4) and 2016/17 (wave 8) were analysed. Serum levels of inflammatory markers (C-reactive protein (CRP)) and covariates (age, sex, education, wealth, body mass index, smoking, cholesterol, triglyceride) were measured at wave 4 (considered here as the baseline). Self-reported weekly moderate/vigorous PA (vs no weekly moderate/vigorous PA) was examined at a four-year follow-up (wave 6, 2012/13). Depressive symptoms were assessed at baseline and six years later (wave 7, 2014/ 15) using the 8 -item version of the Centre for Epidemiological Studies Depression Scale (CES-D). Binary mediation analysis was used to investigate whether PA mediated the relationship between systemic inflammation and depressive symptoms, adjusting for the full set of covariates.

Results No significant associations were found between elevated levels of CRP and subsequent depressive symptoms (Odds Ratio $(\mathrm{OR})=1.28 \quad$ (95\% Confidence Intervals (CI)) 0.98-1.68). Participants with high CRP were significantly more likely to be physically inactive (OR $=1.29$ (95\% CI) 1.07 to 1.56$)$. Physical inactivity was associated with greater 
odds of subsequent depressive symptoms (OR=2.17 (95\% CI) 1.65-2.84). Mediation analyses revealed that physical inactivity mediated the relationship between high CRP and depressive symptoms, explaining a total of $47.79 \%$ of this association.

Conclusion In this nationally representative sample, we found that physical inactivity is a partial mediator of the relationship between high CRP and subsequent depressive symptoms. Interventions targeting physical inactivity may be effective in ameliorating inflammation-associated depressive symptoms.

\section{OP62 A POPULATION BASED STUDY: ASSESSING MALE SUICIDE TRENDS AND INEQUALITIES IN SCOTLAND 1980-2015}

OR Molaodi*, D Brown, R Dundas, AH Leyland. MRC/CSO Social and Public Health Sciences Unit, University of Glasgow, Glasgow, UK

\subsection{6/jech-2018-SSMabstracts.61}

Background Male suicide rates increased remarkably in Scotland in the 1980s and 1990s, with higher rates in more deprived areas. We examine trends in male suicide in Scotland from 1980 to 2015 by method of suicide and individual socioeconomic position. We also explore trends in inequalities by individual socioeconomic position and the extent to which this may be attributable to suicide method.

Methods Suicide deaths for 1980-2015 were obtained from National Records Scotland. Inequalities were assessed using National Statistics socio-economic classification (NS-SEC) for ages 20-59 in 2000-2002 and 2010-2012. Standardised death rates were calculated by age with European population 2013 as reference, and Poisson regression was used to assess trend significance. Inequalities were assessed using Slope of Index of Inequality (SII).

Results There were 12281 suicide deaths between 1980 and 2015. No significant linear trend was observed over time for all ages, but suicide rates per 100000 person-years increased from 21 to 27 between 1980 and $2002 \quad(\mathrm{p}<0.001)$, and decreased from 27 to 20 from 2002 to 2015 ( $p<0.001)$. No significant trend was observed for poisoning, but suicide rate by hanging, suffocation and strangulation increased over time $(p<0.001)$, and decreased for other suicide $(p<0.001)$. There were significant differences in suicide rates between the NSSEC groups, and by suicide method (p-values $<0.001)$. SII: 85 (95\% CI, 77 to 92 ) in 2000-01 and 62 (95\% CI, 55 to 68) in 2010-12 per 100000 person-years, indicated that inequalities between social class extremes were significantly higher in 2000-02 than 2010-12. Suicide by hanging, suffocation and strangulation accounted for 44\% of inequalitiesin 2000-02 $(\mathrm{SII}=37 \quad(95 \% \mathrm{CI}, 33$ to 42$))$ and $49 \%$ in $2010-2012$ $(\mathrm{SII}=30(95 \% \mathrm{CI}, 25$ to 34$))$.

Conclusion The decline in male suicide rates may be attributed to suicide prevention strategies introduced by the Scottish Government from 2002 such as Choose Life. Despite decreasing trends of male suicide, suicide by hanging, suffocation, and strangulation increased over time. Inequalities by individual deprivation decreased between 2000-02 and 2010-12. Limitations are that NS-SEC categories, never worked and long term unemployed and not classified, were excluded from the analysis assessing inequalities since the interpretation of results for this group was ambiguous. What effect this omission would have on the estimation of inequalities is unclear. Caution should be taken when comparing inequalities by NSSEC 2001 and 2011 as in 2011 NS-SEC category was estimated for those without occupation. Policy should be directed at reducing deaths from hanging, suffocation, and strangulation.

\section{OP63 LONELINESS, LIVING ARRANGEMENTS AND EMOTIONAL SUPPORT AS PREDICTORS OF SUICIDALITY: A 7 YEAR FOLLOW-UP OF THE UK BIOBANK COHORT}

RJ Shaw*, B Cullen, N Graham, D Mackay, J Ward, R Pearsall, DJ Smith. Institute of Health and Wellbeing, University of Glasgow, Glasgow, UK

\subsection{6/jech-2018-SSMabstracts.62}

Background Between 1997 and 2017 the number of middleaged people living alone in the UK increased by $53 \%$ and loneliness is now recognised as an important policy area. We aimed to understand the interrelationships between loneliness, living arrangements and emotional support in predicting suicidal thoughts and behaviours.

Methods Between 2006 and 2010 sociodemographic and health data were collected from 500000 participants, aged 40-69, in UK Biobank. These data were linked to hospital admission records for self-harm and suicidal ideation until March 2015, and records for death by suicide until February 2016. Additionally, in 2016-2017, 150000 participants completed an online questionnaire which probed thoughts of selfharm, self-harm behaviour and attempted suicide. Exposures assessed were baseline measures of self-reported loneliness, living arrangements and emotional support (frequency of confiding). Deaths by suicide and hospital admissions were investigated with Cox proportional hazards models and logistic regression was used for self-report outcomes. Analyses were adjusted for socio-demographic factors including deprivation and employment, and multimorbidity.

Results In adjusted analyses loneliness was the risk most consistently related to all outcomes including death by suicide, (hazard ratio (HR) $1.75,95 \%$ CI 1.22 to 2.51), hospital admissions (HR 4.41, 95\% CI 2.50 to 7.76 ) and self-reported suicide attempts (HR 5.38, 95\% CI 3.35 to 8.63). After adjustment, not living with a partner was associated with increased risks of hospital admissions and dying by suicide, but not with the self-report measures of suicidality. Not living with a partner had a stronger relationship with death by suicide for men (HR 2.08, 95\% CI 1.36 to 3.18) than for women (HR 1.16, 95\% CI 0.59 to 2.31). After adjustment, emotional support was associated with the self-report but not administrative measures of suicidality. For example, when people with the least emotional support were compared to those with the most, the odds ratio was 3.00 (95\% CI 1.71 to 5.28). Statistical interactions indicated that people who were lonely despite living with a partner had the highest risk of contemplating self-harm and that loneliness eliminated the protective effects of living with a partner for deaths by suicide.

Conclusion Loneliness was the strongest predictor of suicide risk irrespective of living arrangements and emotional support and loneliness explained the relationships between living 